\title{
Probing parsec scale jets in AGN with geodetic VLBI
}

\author{
Alexander B. Pushkarev** \\ Max-Planck-Institut für Radioastronomie, Auf dem Hügel 69, 53123 Bonn, Germany \\ Pulkovo Observatory, Pulkovskoe Chaussee 65/1, St. Petersburg 196140, Russia \\ Crimean Astrophysical Observatory, Nauchny 98409, Crimea, Ukraine \\ E-mail: apushkar@mpifr-bonn.mpg.de

\section{Yuri Y. Kovalev} \\ Max-Planck-Institut für Radioastronomie, Auf dem Hügel 69, 53123 Bonn, Germany \\ Astro Space Center of Lebedev Physical Institute, Profsoyuznaya 84/32, Moscow 117997, Russia \\ E-mail: ykovalev@mpifr-bonn.mpg.de
}

\begin{abstract}
We report on an ongoing effort to image active galactic nuclei simultaneously observed at 2.3 and 8.6 GHz in the framework of a long-term VLBI project RDV (Research \& Development - VLBA) started in 1994 aiming to observe compact extragalactic radio sources in the astrometric/geodetic mode. Observations of bright extragalactic sources are carried out bi-monthly making up to six sessions per year with participation of all ten VLBA antennas and up to nine additional (geodetic and $\mathrm{EVN}$ ) radio telescopes. Analysis of single-epoch results for 370 quasars, BL Lacs and radio galaxies is presented. We discuss VLBI core properties (flux densities, sizes, brightness temperatures); spectral characteristics of the cores and jets; evolution of brightness temperatures in the jets.
\end{abstract}

The 9th European VLBI Network Symposium on The role of VLBI in the Golden Age for Radio Astronomy and EVN Users Meeting

September 23-26, 2008

Bologna, Italy

\footnotetext{
* Speaker.

†cknowledgements. This work is based on the analysis of global VLBI observations including the VLBA, the raw data for which were provided to us by the NRAO archive. The National Radio Astronomy Observatory is a facility of the National Science Foundation operated under cooperative agreement by Associated Universities, Inc. This research has made use of the NASA/IPAC Extragalactic Database (NED) which is operated by the Jet Propulsion Laboratory, California Institute of Technology, under contract with National Aeronautics and Space Administration. Y.Y. Kovalev is a Research Fellow of the Alexander von Humboldt Foundation.
} 


\section{Introduction}

A long-term VLBI project RDV (Research \& Development - VLBA) aimed at observations of bright compact extragalactic radio sources was started in 1994 under coordination of NASA and NRAO [1]. The simultaneous observations at 2.3 and $8.6 \mathrm{GHz}$ are carried out bi-monthly making up to five or six sessions per year with participation of all ten 25-m VLBA antennas and up to nine geodetic and EVN stations. The participation of the southern antennas such as HartRAO (South Africa) and TIGO (Chile) allowed us to successfully observe sources with declination up to $-47^{\circ}$. The sample of observing objects consists of $\sim 500$ sources. It is important to note that the sample is not flux density complete. In each experiment 80-90 active galactic nuclei are observed and $\sim 50$ objects form a core of the sample and are scheduled continuously. The sample is dominated by quasars, with the weak-lined BL Lacs and radio galaxies making up $8.3 \%$ and $7.8 \%$ of the sample, respectively.

\section{Data Reduction}

The data were correlated at the VLBA correlator in Socorro, with a 4 sec integration time, and were obtained by us from the public NRAO archive and then calibrated in AIPS using techniques adopted for subarrayed data sets. System temperatures and values of SEFD measured during the observations were used for the initial amplitude calibration. Using well measured gains for the VLBA antennas we were able to improve the amplitude calibration for non-VLBA stations applying self-calibration. We estimate the accuracy of amplitude calibration to be better than $10 \%$. Phase corrections for residual delays and delay rates were done using the AIPS task FRING applying a point-like source model. Self-calibration, hybrid mapping, and model fitting were performed in DIFMAP. In the model fitting, we used a minimum number of circular Gaussian components that was reproducing adequately the observed interferometric visibilities.

\section{Results and Discussion}

We discuss first-epoch results for 370 active galactic nuclei (Fig. 1, left) on the basis of high dynamical range $(\sim 1000)$ images obtained at 2.3 and $8.6 \mathrm{GHz}$. The sources are bright (in $92 \%$ the
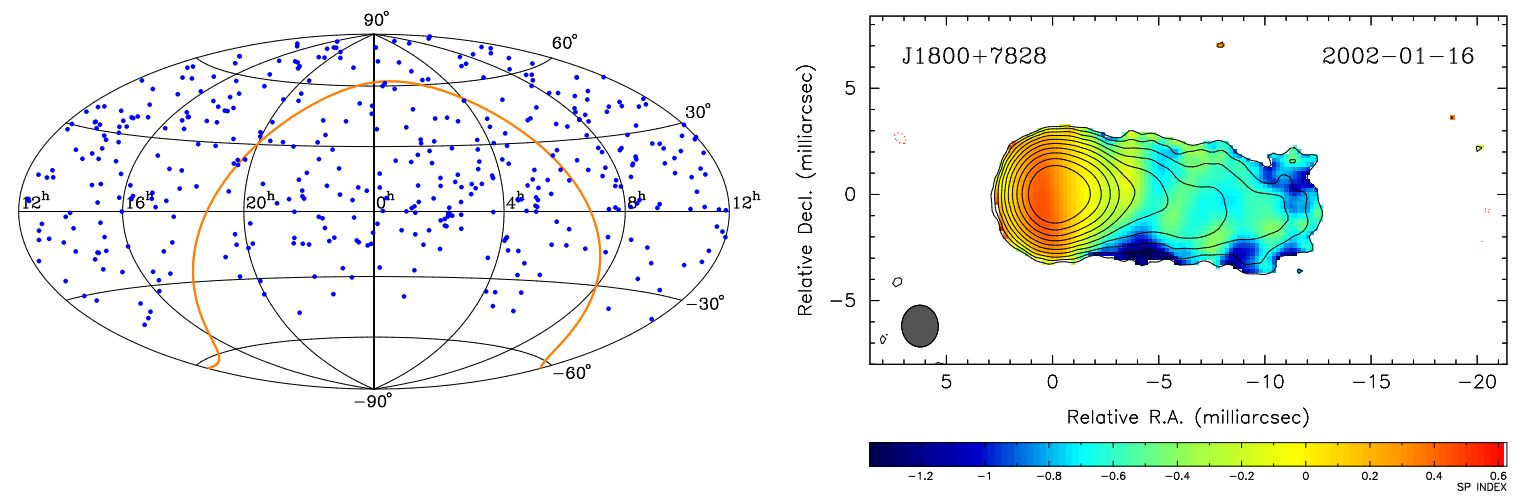

Figure 1: Sky distribution for the 370 sources (left). Spectral index distribution in J1800+7828 calculated between 2.3 and $8.6 \mathrm{GHz}$ with the $8.6 \mathrm{GHz}$ total intensity contours superimposed (right). 

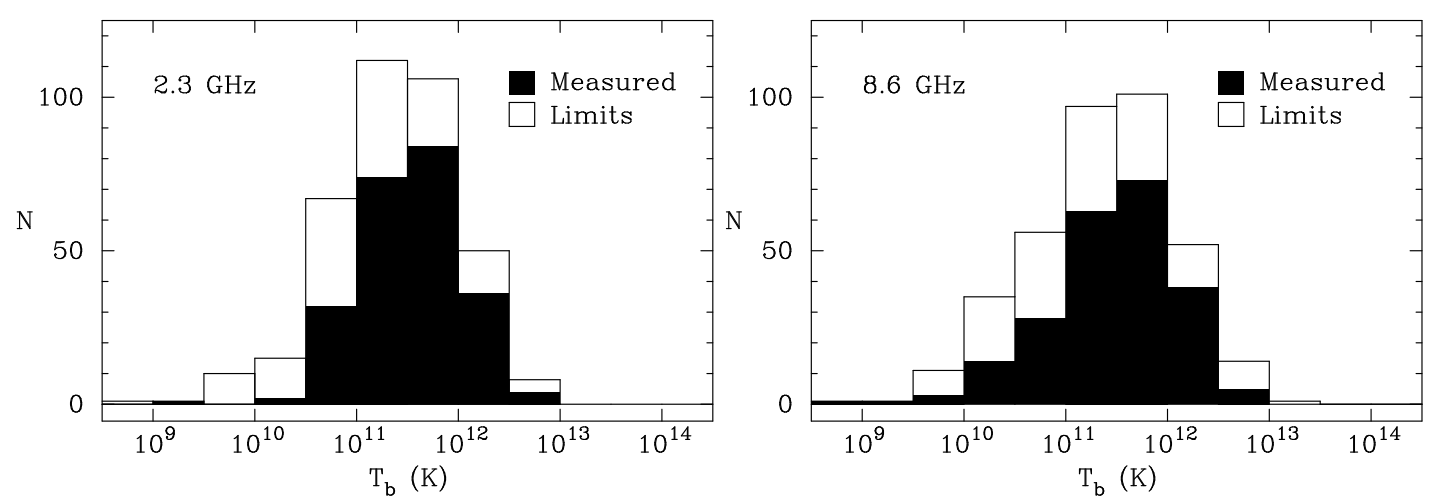

Figure 2: Brightness temperature histograms for the core components at $2.3 \mathrm{GHz}$ (left) and $8.6 \mathrm{GHz}$ (right).

correlated flux density was more than $200 \mathrm{mJy}$ ). One half of the sources are compact and coredominated (VLBI compactness greater than 0.51 , core dominance greater than 0.75 ). The median values of the core size are 0.28 and 1.04 mas at 8.6 and $2.3 \mathrm{GHz}$, respectively.

Dual-frequency VLBI observations provide a possibility to study spectral properties on parsec scales. In Fig. 1 (right) we plot the spectral index distribution map for J1800+7828 as a typical example. Most of the VLBI cores have flat spectra $\left(\alpha_{\text {core }} \sim 0, S \propto v^{\alpha}\right)$ since the radiation from these regions is dominated by optically thin emission from the jet base. We have cross-identified 48 jet components in 38 sources, the median value of the obtained spectral indices is $\alpha_{\text {jet }}=-0.75$ indicating optically thin radiation. This results in the median value of the power index energy distribution of radiative particles to be 2.5. The determination of the spectral index of the jet components has been done taking into account a correction for the respective core shifts found in these sources [2].

We have also measured the core brightness temperatures in the source rest frame. The respective distributions at 2 and $8 \mathrm{GHz}$ shown in Fig. 2 have close median values of $2.5 \times 10^{11} \mathrm{~K}$. The empty bins are the lower limits and represent the cases when either the source has unknown redshift or an upper resolution limit has been used for the size of the component.

The sources with the prominent and well modeled jets having at least three jet components at both frequencies were the cases of our particular interest, since they provided us with information about brightness temperature evolution along the jets as a function of a distance to the core, $r$. The brightness temperature gradients can be well fitted with power law $T_{\mathrm{b}} \propto r^{-k}$ for 12 selected sources. The power index $k$ varies with values typically between 1.2 and 3.6 with the median value of $k=2$. Applying synchrotron radiation theory for a conical jet model $[3,4]$ and taking into account the median values for the jet spectral index $\alpha=-0.75$ and power law index $k=2$ we obtained the dependencies of electron density $n_{e} \propto r^{-1.5}$ and magnetic field $B \propto r^{-0.9}$ along the jet.

\section{References}

[1] L. Petrov, D. Gordon, J. Gipson, et al., Journal of Geodesy, in press (2009); astro-ph/0806.0167.

[2] Y.Y. Kovalev, A.P. Lobanov, A.B. Pushkarev, and J.A. Zensus, A\&A 483, 759 (2008).

[3] A.P. Lobanov, $A \& A$ 330, 79 (1998).

[4] M. Kadler, PhD thesis, Bonn University (2005). 